\title{
Prof. Dr. Gertrude Lübbe-Wolff, Richterin des Bundesverfassungsgerichts
}

\author{
Juristinnen machen Karriere - wir stellen sie vor
}

\begin{abstract}
Die Fragen stellte Louisa Bartel, Richterin am Oberlandesgericht Karlsruhe und Mitglied der Regionalgruppe Karlsruhe im September 2009 in Karlsruhe.
\end{abstract}

Sie können schon heute auf eine außergewöhnlich erfolgreiche juristische Karriere zurückblicken - welchen Berufswunsch hegten Sie als Schülerin?

Ich wollte schon als Schülerin, nachdem die Puppenspielund die Indianerspielphase vorbei waren, am liebsten Professorin werden. Ursprünglich für Philosophie.

Warum haben Sie sich dann für das Studium der Rechtswissenschaften entschieden?

Ein bisschen Philosophie habe ich ja auch studiert. Aber nur das zu machen, davon hatte mir mein Vater mit einem Argument abgeraten, das ich damals sehr einleuchtend fand: Als Philosoph müsse man erst einmal etwas haben, worüber man philosophieren kann, also eine außerphilosophische Sachkenntnis. Dann habe ich geschwankt, ob ich Jura, Mathematik oder Linguistik studieren sollte. Mathematik habe ich schließlich trotz guten Zuredens meiner Mathelehrerin ausgeschlossen, weil ich mir nicht sicher war, ob ich das wirklich gut kann. Ich war bloß gut im Beweisen, beim Kopfrechnen habe ich immer Fehler gemacht. Um zwischen Linguistik und Jura zu entscheiden, habe ich mir in jedem dieser Fächer eine Vorlesungsstunde angehört. Bei den Linguisten wurden in dieser Stunde gerade nur Vokale und Konsonanten gezählt. In der juristischen Vorlesung stritt man sich über den Schweinemästerei-Fall, das fand ich interessanter.

\section{Was reizte Sie an der akademischen Tätigkeit?}

Das Denken und die Freiheit.

Sie sind 1974 in die USA gegangen - warum die USA und warum die Harvard Law School?

Sorgfältig geplant war das aus heutiger Sicht nicht, ich habe mich einfach für die Uni entschieden, deren Name damals in meinen Ohren den besten Klang hatte, ohne dass ich sonst viel darüber gewusst hätte. Die Professoren und die Mitstudenten und das ganze Programm waren dann wirklich sehr gut, und ich habe mich ohnehin mit ganz anderen Sachen beschäftigt als ursprünglich beabsichtigt.

Womit haben Sie sich dort beschäftigt und welche Erfahrungen waren für Sie - persönlich und beruflich - besonders wertvoll?

Studiert habe ich dort hauptsächlich Völkerrecht, von dem ich in Deutschland im Studium überhaupt nichts mitbe-
Gertrude Lübbe-Wolff wurde 1953 in Weitensfeld (Kärnten) geboren und studierte nach dem Abitur Rechtswissenschaften an den Universitäten Bielefeld, Freiburg im Breisgau und an der Harvard Law School in Cambridge/ Massachusetts. Nach dem Zweiten Juristischen Staatsexamen 1977 und dem Abschluss der Dissertation bei Prof. Dr. Alexander H. Hollerbach mit der Arbeit „Rechtsfolgen und Realfolgen“ war sie von 1979 bis 1987 zunächst als wissenschaftliche Mitarbeiterin und später als Hochschulassistentin an der Universität Bielefeld tätig. 1987 habilitierte sie sich bei Professor Dr. Dieter G. Grimm mit der Arbeit „Die Grundrechte als Eingriffsabwehrrechte“ und erhielt die venia legendi für Öffentliches Recht, Verfassungsgeschichte der Neuzeit und Rechtsphilosophie. 1988 übernahm sie die Leitung des Umweltamts der Stadt Bielefeld. Zum Sommersemester 1992 wechselte sie an die Universität Bielefeld und übernahm einen Lehrstuhl für Öffentliches Recht. Im Zeitraum von 1996 bis 2000 war sie stellvertretendes Wahlmitglied des Verfassungsgerichtshofs Nordrhein-Westfalen und von 1996 bis 2002 außerdem Geschäftsführende Direktorin des Zentrums für interdisziplinäre Forschung (ZiF) der Universität Bielefeld. Im Jahr 1999 erhielt sie den höchstdotierten deutschen Forschungspreis, den Gottfried Wilhelm Leibniz-Preis, und übernahm im Jahr 2000 den Vorsitz im Sachverständigenrat für Umweltfragen. Im April 2002 wurde sie zur Richterin des Bundesverfassungsgerichts gewählt und gehört dort dem für das Staatsorganisationsrecht zuständigen Zweiten Senat an.

Frau Lübbe-Wolff ist verheiratet und hat vier erwachsene Kinder.

kommen hatte, außerdem amerikanisches Verfassungsrecht und alles mögliche andere. Das Wichtigste von allem war aber einfach das Leben in einem anderen Land, der Umgang mit so vielen Menschen aus allen Erdteilen, und die damit verbundene Erfahrung der Nicht-Selbstverständlichkeit von so Vielem, was ich bis dahin an Meinungen und Gegebenheiten für selbstverständlich gehalten hatte. Diese Erfahrung wünsche ich jedem.

Das hört sich so an, als ob Sie Karriereplanung im eigentlichen Sinne eher nicht betrieben haben?

Das Planvolle bestand hauptsächlich darin, dass ich die notwendigen Qualifikationsarbeiten geschrieben habe. Gezielt Kontakte knüpfen, netzwerken, möglichst viel Konferenzpräsenz zeigen, wie es heute jungen Wissenschaftlern empfohlen wird - auf den Gedanken wäre ich gar nicht ge- 
kommen. Ich habe einfach gern und viel gearbeitet. Weil ich diese Arbeit wollte, wollte ich ja Professorin werden.

Ist Karriereplanung und „Netzwerken“ - Dinge, die Männer angeblich so viel besser beherrschen als Frauen - also überflüssig?

Ich würde eher sagen, das ist eine Frage des richtigen Maßes. Bei mir lag vielleicht ein Fall von Untermaß vor. Wieviel tatsächlich über persönliche Kontakte läuft, das habe ich mir damals gar nicht klargemacht. Aber diese $\mathrm{Na}$ ivität hatte für mich eben die gute Seite, dass ich nicht in der gefährlichen Versuchung war, über irgendwelchem kurzatmigen Showgeschäft die eigentliche Arbeit zu vernachlässigen. in der Schweiz inne, ihre jüngeren Schwestern Weyma und Anna Lübbe sind gleichfalls Professorinnen - welche Anregungen verdanken Sie Ihrem Elternhaus? Hat Ihr Vater Ihre Entscheidung, eine wissenschaftliche Laufbahn einzuschlagen, beeinflusst?

Auf jeden Fall. Aber überhaupt nicht auf direkte Weise, sondern durch die Rolle, die er in der Familie spielte. Außerdem wurde zuhause ständig gelesen und über irgendwelche Sachthemen diskutiert.

Welche Bedeutung hatte die Studienstiftung des Deutschen Volkes für Ihre berufliche Entwicklung?

Von der Studienstiftung habe ich aus der Zeit, als ich in Deutschland studierte, vor allem die sehr schönen und inte-

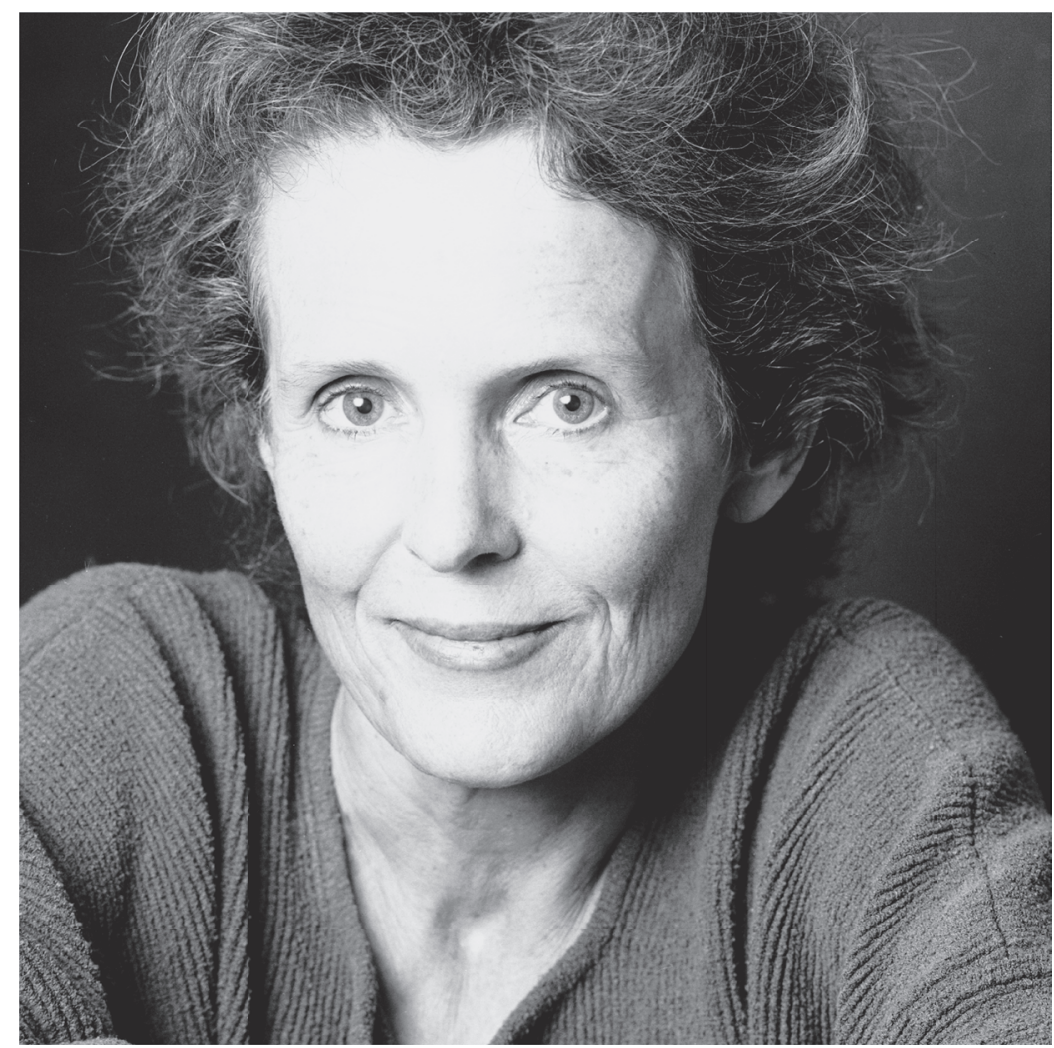

\section{Hatten Sie Vorbilder und Förderer?}

Es gab Vorbilder in der Familie und an der Universität. An der Universität hatte ich sehr förderliche Chefs. Sie haben mir zum Beispiel flexible Arbeitszeiten eingeräumt, so dass ich Berufstätigkeit und Familie unter einen Hut bringen konnte. Besonders wichtig, als Vorbild wie als Förderer, war Dieter Grimm, der meine Habilitationsarbeit betreut hat. Er war für mich der beste akademische Lehrer, den ich mir hätte wünschen können, bewunderungswürdig und vollkommen freilassend.

Sie erwähnen Vorbilder in der Familie. Ihr Vater Hermann Lübbe hatte philosophische Lehrstühle in Deutschland und ressanten Sommerakademien in Alpbach in Erinnerung. Am dankbarsten bin ich für das Jahr in den USA, das die Stiftung gefördert hat.

Eine neue Studie des Hochschulinformationssystems (HIS) zur sozialen Herkunft der Stipendiaten in den Begabtenförderungswerken und eine Untersuchung der Studienstiftung sind zu dem Ergebnis gelangt, dass vor allem Kinder aus Akademikerfamilien gefördert werden; handelt es sich um eine unvermeidliche Folge des Leistungsprinzips oder sehen Sie Änderungsbedarf beim Auswahlverfahren?

Das kann ich nicht beurteilen, weil ich nicht genau genug weiß, wie die Auswahlverfahren heute bei den verschiede- 
nen Förderwerken aussehen. Verbesserungsbedarf sehe ich jedenfalls in der schulischen Bildung und in der Frühförderung von Kindern. Den statistischen Startvorteil, den ein Kind aus einer Familie mitbringt, in der Bücher gelesen und gute Gespräche geführt werden und in der Arbeit nicht als das gilt, was vor dem eigentlichen Leben zu erledigen ist, wird keine Bildungspolitik je ganz wettmachen können. Aber von dem Punkt, an dem die Möglichkeiten zur Förderung von Kindern aus weniger günstigen Verhältnissen ausgeschöpft sind, sind wir noch weit entfernt.

Sie sind nach Ihrer Berufung auf einen Lehrstuhl in Bielefeld als dritte Frau in die Vereinigung der Deutschen Staatsrechtslehrer aufgenommen worden; wie ist man Ihnen dort begegnet?

Keine besonderen Vorkommnisse. Ich war nicht oft bei den Tagungen. Der Frauenanteil lag bei ungefähr einem Prozent, als ich aufgenommen wurde. Heute liegt er, schätze ich, bei knapp zehn Prozent. Die Atmosphäre ist lockerer als früher.

Frauen in herausgehobenen Positionen sind in Wissenschaft und Praxis noch immer selten. Warum ist das so?

Die Hauptursache sehe ich darin, dass Frauen es, statistisch gesehen, noch immer wesentlich schwerer haben als Männer, wenn sie Erfolg im Beruf und Kinder haben wollen. Das kann man daran ablesen, dass unter den Frauen in besonders herausgehobenen Positionen der Anteil derer, die zugleich Kinder haben, wesentlich kleiner ist als bei Männern in den entsprechenden Stellungen. Ich habe das einmal bei einer Reihe von Spitzeninstitutionen der Rechtspflege recherchiert für eine Veröffentlichung über Frauen in juristischen Berufen. Das Ergebnis war so, dass ich die präzisen Zahlen zu diesem Punkt gar nicht veröffentlichen konnte, weil das bei der kleinen Zahl der Frauen an den entsprechenden Institutionen auf eine Preisgabe personenbezogener Daten hinausgelaufen wäre. Die Situation wird sich bessern durch alles, was die Vereinbarkeit von Familie und Beruf erleichtert. Diese Vereinbarkeit sollte nicht nur für Frauen gefördert werden, sondern auch für Männer, die Familienarbeit leisten.

Sie haben sich intensiv - wissenschaftlich und in der praktischen Tätigkeit - mit Umweltrecht beschäftigt - was hat Sie an diesem Fachgebiet fasziniert?

Dass es besonders zukunftswichtig ist, dass es viele ganz neue Arten von Rechtsfragen aufwarf und dass es sich so gut zum Studium der Probleme eignet, die bei dem Versuch auftreten, rechtlichen Regeln praktische Wirksamkeit zu verschaffen.

Sie haben sich 1988 für einen Wechsel von der Universität in die Praxis entschieden und die Leitung des neu gegründeten Umweltamtes in Bielefeld übernommen. Was hat Sie dazu bewogen?
Ich bin in die Verwaltung gegangen, weil ich nicht von meiner Familie wegpendeln, sondern in Bielefeld bleiben wollte. Das wäre nicht möglich gewesen, wenn ich mein altes Berufsziel zu diesem Zeitpunkt weiterverfolgt hätte. Außerdem hatte ich auch Lust auf praktische Erfahrungen. Amtsleiterin in einer Kommunalverwaltung, das ist eine extrem lehrreiche Tätigkeit. Ich hatte da ja nicht nur mit Umweltrecht und naturwissenschaftlichen und technischen Umweltfragen zu tun, sondern auch mit Bauordnung und Bauplanung, von dieser Fachseite aus. Dann mit der Führung von 160 Mitarbeitern aller Statusgruppen. Mit dem Haushalt, mit dem Kommunalparlament, mit der Interaktion von Verwaltung, Politik und Wirtschaft. Da hatte man eigentlich den ganzen Kosmos der Demokratie im Kleinen.

Welche der sicher zahllosen beruflichen Erfahrungen, die Sie dort sammeln konnten, waren aus heutiger Sicht besonders wertvoll?

Von dem, was ich an praktischer Klugheit besitze, egal wie viel oder wie wenig es ist, gehen neun Zehntel auf diese Jahre zurück. Das Wichtigste, was ich daraus mitgenommen habe, ist ein besserer Sinn für die Art und Weise, wie Menschen in ihrem Verhalten immer zugleich durch ihren Willen und durch die institutionellen Rahmenbedingungen bestimmt sind, und wie das beides miteinander zusammenhängt.

Das ist wunderbar abstrakt formuliert - könnten Sie uns ein Beispiel geben?

Ich hatte mal das Problem, dass wir zuwenig Probenehmer hatten für die Entnahme von Abwasserproben aus den betrieblichen Kanalnetzen und im Verhältnis dazu zu viele Laboranten. Also wollte ich, dass die Laboranten auch beim Probenehmen mitmachen. Sie glauben nicht, wie schwierig das war. Immer neue Berge von Sicherheitsproblemen. Man wollte Sicherheitsanzüge wegen der giftigen Chemikalien, die im Kanal sein können, ein Ex-Meter zum Messen, ob Explosionsgefährliches dabei ist, spitzenverstärkte Sicherheitsschuhe für den Fall, dass einem beim Kanaldeckelheben das Ding auf die Füße fällt, rotweiße Absperreinrichtungen, weil die Schächte auf innerbetrieblichen Fahrwegen liegen können, einen Dienstbulli, weil die ganze Montur sich nicht mehr mit einem normalen PKW transportieren lässt, eine Abdeckung, damit man beim Probenehmen nicht in den Kanalschacht fällt, und was nicht noch alles. Der eingeschaltete Sicherheitsbeauftragte nickte nur. Wochenlanges Hin und Her mit immer neuen Anforderungen, die erst noch erfüllt werden mussten, es ging einfach nicht voran. Schließlich kam ich, reichlich spät, auf den Gedanken, dass wir es vielleicht gar nicht mit einem Sicherheitsproblem zu tun hatten, sondern die Leute sich Sorge machten, sie würden tariflich heruntergruppiert, wenn sie auch solche niedriger eingestufte Arbeit machten. Das Thema wurde angesprochen, ich verbürgte mich dafür, dass das 
nicht passieren würde, und siehe da, alle Sicherheitsprobleme wie weggeblasen, am nächsten Tag ging es los mit dem Probenehmen, und die schöne Konstruktion zur Verhinderung von Kanalstürzen, die ein hilfreicher Abteilungsleiter wochenends zuhause geschreinert hatte, kam nie zum Einsatz.

\section{Warum sind Sie an die Universität zurückgegangen? Haben} Sie das wissenschaftliche Arbeiten vermisst?

Ich hätte mir damals sehr gut vorstellen können, in der Praxis zu bleiben, aber dann gab es eine passende offene Stelle ausgerechnet an derselben Universität, an der auch mein Mann tätig war. Dieses große Los musste ich dann doch zu ziehen versuchen.

Sie sind seit nunmehr sieben Jahren Richterin des Bundesverfassungsgerichts. Das Gericht genießt in der Bevölkerung hohes Ansehen und Vertrauen. Worin sehen Sie die Gründe für diesen erfreulichen Befund?

Der wichtigste Grund für das hohe Vertrauen, das das Gericht genießt, ist die Institution der Individualverfassungsbeschwerde, die jeder Bürger ohne große Hürden erheben kann. Ohne diese Möglichkeit könnten wir noch so gute Arbeit machen, es würde dem Gericht keine vergleichbare Bekanntheit, Vertrauensstellung und Autorität daraus zuwachsen. So aber wird immer wieder deutlich, was das Gericht und was die Verfassung, nach der es Recht spricht, für jeden Bürger und für jeden, der sich in Deutschland aufhält, bedeuten. Die wichtigste Folge davon ist nicht das hohe Ansehen des Gerichts, sondern das hohe Ansehen des Grundgesetzes.

Rudolf Smend hat einmal - in einer Festrede anlässlich des zehnjährigen Bestehens des Gerichts im Jahr 1962 - gesagt: „Das Grundgesetz gilt nunmehr praktisch so, wie das Bundesverfassungsgericht es auslegt [...]“. Ist das eine zutreffende Analyse?

Ja. Dass die Verfassung so gilt, wie das Verfassungsgericht sie auslegt, und nicht so, wie der Bund sie auslegt, wenn er den Ländern Kompetenzen streitig macht, oder wie eine Bundestagsmehrheit sie auslegt, wenn sie die Arbeitsmöglichkeiten der Opposition verschlechtern will, das ist ja der Sinn einer Verfassungsgerichtsbarkeit.

Sie betreuen als Berichterstatterin unter anderem Verfassungsbeschwerden, die den Vollzug von Straf- und Untersuchungshaft sowie den Maßregelvollzug betreffen und sind damit auf einem Rechtsgebiet tätig, das vorher nicht zu Ihren Forschungsschwerpunkten zählte. Wie haben Sie sich in diese Spezialmaterie eingearbeitet?

Das Verfassungsgericht ist ja nicht für die Auslegung und Anwendung des Strafvollzugsrechts, sondern für die Auslegung und Anwendung des Verfassungsrechts zuständig. Man muss aber natürlich das Rechtsgebiet kennen, auf das unsere Entscheidungen sich beziehen und auf das sie ein- wirken. Dazu habe ich Bücher gelesen, die bisherige Rechtsprechung des Bundesverfassungsgerichts auf diesem Gebiet studiert und eine entsprechende geordnete Datei angelegt, Strafvollzugsanstalten besucht, dort mit Leitungspersonen, anderen Bediensteten und Gefangenenvertretern gesprochen. Außerdem habe ich immer meine Mitarbeiter so ausgesucht, dass welche mit einschlägiger praktischer Erfahrung dabei waren.

Welche Eindrücke haben Sie von der Wirklichkeit des Haftvollzugs gewonnen? Haben Sie in den vergangenen Jahren Anzeichen für Veränderungen hin zu einer restriktiveren Vollzugspraxis wahrgenommen?

Es gab in den zurückliegenden Jahren in vielen Bundesländern eine deutliche Tendenz zu einer restriktiveren Praxis. Das hängt auch mit Personal- und Geldmangel zusammen. Das Niveau an Rechtsstaatlichkeit ist im deutschen Strafvollzug nach meinem Eindruck im allgemeinen hoch, aber Behandlungsangebote und die Unterbringungssituation der Gefangenen sind oft unzureichend. Dasselbe gilt für den Schutz der Gefangenen vor wechselseitiger Unterdrückung und Gewalt. Dafür spielt die Unterbringungssituation und die Personallage eine wichtige Rolle. Denken Sie an den entsetzlichen Siegburger Fall, in dem drei Haftraumbewohner einen vierten zu Tode gefoltert haben. Das wäre nicht möglich gewesen, wenn die Hafträume nur mit einem Gefangenen belegt gewesen wären, oder auch mal mit zwei richtig zusammengestellten, und wenn genug Personal dagewesen wäre, dass man die vier nicht rund um die Uhr zusammen hätte einschließen müssen, ohne dass jemand Zeit zum genauen Hinsehen hat.

Empfinden Sie die Arbeit „am Fall“ mit den damit einhergehenden Beschränkungen als Bürde oder als Bereicherung?

Eindeutig als Bereicherung, auch wenn die Menge der Arbeit manchmal ganz schön belastet.

In den vergangenen Jahren sind die Zahl der Verfassungsrichterinnen und die Zahl der wissenschaftlichen Mitarbeiterinnen am Gericht gesunken - woran liegt das?

Jedenfalls nicht daran, dass es keine geeigneten Frauen gegeben hätte. Wenn der Frauenanteil unter den Verfassungsrichtern sinkt, hat das vermutlich automatisch auch ein Absinken des Frauenanteils bei den wissenschaftlichen Mitarbeitern zur Folge. Mir haben die Landesjustizministerien, bei denen ich nachfrage, wenn ich Mitarbeiter aus dem Bereich der Strafjustiz brauche, meist Frauen vorgeschlagen. Ich nehme an, dass das anders läuft, wenn die Anfrage von einem Mann kommt. Dass Frauen bei den wissenschaftlichen Mitarbeitern nach wie vor erheblich unterrepräsentiert sind, liegt übrigens auch daran, dass Frauen häufiger familienhalber Probleme mit dem Umzug oder der Pendelexistenz haben, die mit einer Abordnung an einen anderen Dienstort verbunden sind. Man sieht das daran, dass bei 
den Mitarbeiterinnen der Anteil derer, die keine Familie mit Kindern haben, wesentlich größer ist als bei ihren männlichen Kollegen.

Sie haben - wie alle Verfassungsrichter - jeweils vier wissenschaftliche Mitarbeiter, die Sie bei Ihrer Arbeit unterstützen. Wie hoch ist der Frauenanteil unter Ihren wissenschaftlichen Mitarbeitern, Frau Lübbe-Wolff?

Zur Zeit ist eine Stelle vakant und der Frauenanteil beträgt sechsundsechzigkommaperiodesechs Prozent. Demnächst dann wieder fünfundsiebzig. Es gab auch schon fifty-fiftyPhasen. Ich habe keine Quote, das ist einfach das Ergebnis meines Versuchs, gute Leute zu bekommen, und der schon erwähnten Vorschlagspraxis.

Sie beschäftigen sich auch mit philosophischen und moralischen Fragen. Welcher Philosoph hat Ihr Denken besonders geprägt?

Wenn ich von den Philosophen in meiner Familie mal absehe: Hegel.

Sie haben neben einem anspruchsvollen Berufsleben vier Kinder. Wie ist es Ihnen persönlich gelungen, Familie und Beruf zu vereinbaren?

Mit einem Partner, dem man dieselbe Frage stellen könnte, mit sehr viel Arbeit, und mit sehr viel Glück. Gesunde
Kinder, genug Geld für Mitbetreuung durch Dritte, und Berufe, die uns in der wichtigsten Familienzeit Sesshaftigkeit an einem Ort ermöglicht haben. Dafür habe ich zwar viele sehr verlockende Angebote ausschlagen müssen. Aber ein sehr verlockendes konnte ich dann ja am Ende auch annehmen.

Das ist wohl wahr. Sie sind mit nur 49 Jahren zur Verfassungsrichterin gewählt worden und haben damit alles erreicht, was im Rahmen einer wissenschaftlichen und richterlichen Laufbahn zu erreichen ist. Haben Sie gleichwohl noch Ziele, die Sie verwirklichen wollen, oder Träume?

Mehr als ich werde verwirklichen können. Allein schon für die Bücher, die ich gern noch schreiben würde, wird die verbleibende Lebenszeit wahrscheinlich nicht reichen. Was das Träumen angeht, da stehen nicht Bücher im Vordergrund, sondern Musik.

Ihre vielfältigen beruflichen Verpflichtungen sind kräftezehrend - worin finden Sie einen Ausgleich, um sich zu erholen und „aufzutanken"?

Spazierengehen und Wandern mit Freunden, Schwimmen und, ziemlich dilettantisch, Tennis- und Cellospielen. Und Lesen und Filmegucken, am liebsten so, dass man danach gleich die Nachttischlampe ausmachen kann.

\section{Impressum}

\section{Schriftleitung:}

Anke Gimbal, Rechtsassessorin (V.i.S.d.P.)

Juliane Lindner

Redaktionsanschrift:

Deutscher Juristinnenbund e. V.

Anklamer Str. 38

10115 Berlin

Telefon: 030 443270-0

Telefax: 030 443270-22

E-Mail: geschaeftsstelle@djb.de

\section{Druck und Verlag:}

Nomos Verlagsgesellschaft mbH \& Co. KG

Waldseestr. 3-5

D-76530 Baden-Baden

Telefon: 07221 2104-O

Telefax: 07221 2104-27

\author{
Anzeigenverwaltung und \\ Anzeigenannahme: \\ Sales friendly, Verlagsdienstleistungen \\ Bettina Roos \\ Siegburgerstr. 123 \\ D-53229 Bonn \\ Telefon: 0228 97898-o \\ Telefax: 0228 97898-20 \\ E-Mail: roos@sales-friendly.de
}

Die Zeitschrift sowie alle in ihr enthaltenen einzelnen Beiträge und Abbildungen sind urheberrechtlich geschützt. Jede Verwertung, die nicht ausdrücklich vom Urheberrechtsgesetz zugelassen ist, bedarf der vorherigen Zustimmung des Verlages.

Namentlich gekennzeichnete Artikel müssen nicht die Meinung des Herausgebers oder der Schriftleitung wiedergeben. Unverlangt eingesendete Manuskripte - für die keine Haftung übernommen wird - gelten als Veröffentli- chungsvorschlag zu den Bedingungen des Verlags. Es werden nur unveröffentlichte Originalarbeiten angenommen. Die Verfasser erklären sich mit einer nicht sinnentstellenden redaktionellen Bearbeitung einverstanden.

Erscheinungsweise:

vierteljährlich

Bezugspreis 2009:

jährlich $48,-€$, Einzelheft $13,-€$

Alle Preise zzgl. Vertriebs-Direktbeordnungsgebühren inkl. MWSt.;

Bestellungen nehmen entgegen:

Der Buchhandel und der Verlag; Kündigung jeweils drei Monate zum Kalenderjahresende. Zahlungen jeweils im Voraus an: Nomos Verlagsgesellschaft, Postbank Karlsruhe, Konto 7363651 (BLZ 66010075) oder Stadtsparkasse Baden-Baden, Konto 5002266 (BLZ 66250030). 